\title{
Weil's formulae and multiplicity
}

\author{
by Maria Frontczak and Andrzej Miodek (Eódź)
}

\begin{abstract}
The integral representation for the multiplicity of an isolated zero of a holomorphic mapping $f:\left(\mathbb{C}^{n}, 0\right) \rightarrow\left(\mathbb{C}^{n}, 0\right)$ by means of Weil's formulae is obtained.

Introduction. Let $f=\left(f_{1}, \ldots, f_{n}\right): G \rightarrow \mathbb{C}^{n}, n>1$, be a holomorphic mapping defined on some neighbourhood $G$ of $0 \in \mathbb{C}^{n}$, having an isolated zero at this point. Let $\mu_{c}(f)$ be the covering multiplicity of $f$ at the point 0 (see e.g. [9], Ch. V, $§ 2$, Sec. 1). Then there exist arbitrarily small neighbourhoods $\Omega \subset G$ and $\Delta$ of $0 \in \mathbb{C}^{n}$ such that, for almost all $w \in \Delta$ (i.e. outside some proper analytic set), the number $\#\left(f^{-1}(w) \cap \Omega\right)$ of pre-images of $w$ by $f$ lying in $\Omega$ is equal to $\mu_{c}(f)$ (cf. [9], Ch. V, $\S 2$, Proposition).

The integral representation of the above multiplicity is well known and often used (see [6], Ch. V, §1, 2, [10], Ch. IV §18, Sec. 55, (6)). The proofs of this representation are based on the Stokes theorem. But there are some difficulties connected with the choice of a suitable version of this theorem, caused by the occurrence of singular points and by the necessity of integrating over noncompact manifolds. These difficulties are usually passed over in silence.

In this paper we get the integral representation of the multiplicity in full detail. We overcome the difficulties mentioned by applying some properties of totally real manifolds (see $\S 2$ ) and Weil's formula obtained in [5] with the use of a multivalued mapping and without using the Stokes theorem (see $\S 3,(1),(3))$.
\end{abstract}

1. Notations and basic notions. We adopt the definitions of real and complex manifolds in $\mathbb{R}^{n}$ and $\mathbb{C}^{n}$, respectively, from [11], App. II, Def. 4D. Moreover, a real manifold will always be a manifold of class $C^{\infty}$. We assume that such a manifold is equipped with the induced metric.

Let $N \subset \mathbb{C}^{n}$ be a $k$-dimensional complex manifold. Then $N$ is a real manifold of dimension $2 k$. Moreover, for any $p \in N$, the tangent space to

1991 Mathematics Subject Classification: Primary 32A25. 
$N$ at $p$ when $N$ is treated as a real manifold is identical with the tangent space to $N$ at $p$ when $N$ is treated as a complex manifold (cf. [11], App. II, Lemma $5 \mathrm{C})$.

On a real manifold $M$ in $\mathbb{R}^{m}$ or $\mathbb{C}^{n}$ we shall consider the $k$-dimensional Hausdorff measure $\mathcal{H}_{k}, k \leq \operatorname{dim} M$ (cf. [3], Sec. 2.10.2) and the Lebesgue measure $\mathcal{L}$. We shall use the following simple property of these measures (cf. [3], Sec. 2.10.2, [1], App., Sec. 6.1).

Proposition 1. If $k<n=\operatorname{dim} M$, then $\mathcal{H}_{n}(E)=\mathcal{L}(E)=0$ for any set $E \subset M$ such that $\mathcal{H}_{k}(E)<\infty$.

2. Totally real manifolds in $\mathbb{C}^{n}$. Let $L \subset \mathbb{C}^{n}$ be a linear subspace over $\mathbb{R}$. If $L \cap i L=\{0\}$, then $L$ is called a totally real subspace of $\mathbb{C}^{n}$ (cf. [1], App., Sec. 2.4).

Let $M \subset \mathbb{C}^{n}$ be a real manifold. We call $M$ totally real if, for any $p \in M$, the tangent space $T_{p} M$ is a totally real subspace of $\mathbb{C}^{n}$ (cf. [1], App., Sec. 2.4).

Proposition 2. Let $M \subset \mathbb{C}^{n}$ be an $n$-dimensional totally real manifold and $N \subset \mathbb{C}^{n}$ a complex manifold of complex dimension $k<n$. Then $M \cap N$ is a border set in $M$.

Proof. Suppose to the contrary that there exists $U$ open in $M$ such that $U \subset M \cap N$. Then $U$ is an $n$-dimensional real manifold, $N$ is a $2 k$ dimensional real manifold, and $T_{p} U \subset T_{p} N$ for any $p \in U$.

Obviously, $U$ is totally real. Hence if $v_{1}, \ldots, v_{n}$ form a basis of $T_{p} U$, then they are also linearly independent over $\mathbb{C}$. Indeed, if $v_{n}=c_{1} v_{1}+$ $\ldots+c_{n-1} v_{n-1}$ for some $c_{j}=a_{j}+i b_{j}, 1 \leq j \leq n-1$, then $v_{n}=\left(a_{1} v_{1}+\right.$ $\left.\ldots+a_{n-1} v_{n-1}\right)+i\left(b_{1} v_{1}+\ldots+b_{n-1} v_{n-1}\right)$, so $i\left(b_{1} v_{1}+\ldots+b_{n-1} v_{n-1}\right)=$ $v_{n}-\left(a_{1} v_{1}+\ldots+a_{n-1} v_{n-1}\right) \in T_{p} U$, which is impossible because $b_{1} v_{1}+$ $\ldots+b_{n-1} v_{n-1} \in T_{p} U$. This implies that $v_{1}, \ldots, v_{n}, i v_{1}, \ldots, i v_{n}$ are linearly independent over $\mathbb{R}$. Since, clearly, they belong to $T_{p} N$, the real dimension of $T_{p} N$ is at least $2 n$. So $2 k \geq 2 n$, contrary to our assumption. This ends the proof.

Now, we consider the intersection of an analytic set and a totally real manifold.

Let $V$ be an analytic set in some open set $U \subset \mathbb{C}^{n}$. Assume that $\operatorname{dim} V=$ $r<n$. Then we may represent $V$ as the disjoint union

$$
V=N^{r} \cup \ldots \cup N^{0} \text {. }
$$

where each $N^{k}$ is either void or a complex manifold of dimension $k$ (cf. [11], Ch. III, Th. 6G).

Moreover, if $N^{k} \neq \emptyset$, then the closure of $N^{k}$ in $U$ is a locally finite union of irreducible analytic subsets of $V$ whose regular points form $N^{k}$ (cf. [11], 
Ch. III, Th. 1G). Hence $\bar{N}^{k} \cap U$ is an analytic set in $U$ of pure dimension $k$ (cf. [11], Ch. II, Lemma 1I).

Next, let $M \subset U$ be a compact totally real manifold of dimension $n$.

Proposition 3. Under the above assumptions, $V \cap M$ is nowhere dense in $M$.

Pr o of. Since $V$ is closed in $U$ (cf. [11], Ch. II, §1, Prop. (c)) and $M \subset U$ is compact, $V \cap M$ is closed in $M$. By the previous considerations, $V \cap M=$ $\left(N^{r} \cup N^{r-1} \cup \ldots \cup N^{0}\right) \cap M=\left(N^{r} \cap M\right) \cup\left(\left(N^{r-1} \cup \ldots \cup N^{0}\right) \cap M\right)$. In virtue of Proposition 2, $N^{r} \cap M$ is border in $M$. The set $N^{r-1} \cup \ldots \cup N^{0}=V \backslash N^{r}$ is analytic in $U$ (cf. [11], Ch. III, Th. 6F). Therefore $\left(N^{r-1} \cup \ldots \cup N^{0}\right) \cap M$ is closed. As before, it is the union of a border set, $N^{r-1} \cap M$, and a closed set, $\left(N^{r-2} \cup \ldots \cup N^{0}\right) \cap M$. Repeating this argument, we conclude that $\left(N^{1} \cup N^{0}\right) \cap M$ is closed and it is the union of the border set $N^{1} \cap M$ and the set $N^{0} \cap M$ which is nowhere dense in $M$. Thus $\left(N^{1} \cup N^{0}\right) \cap M$ is border in $M$ (cf. [2], Ch. I, Probl. 1.3.E) and, being closed, it is nowhere dense in $M$. Returning to the sets considered at the beginning, we find that $\left(N^{r-1} \cup \ldots \cup N^{0}\right) \cap M$ is nowhere dense in $M$. Hence $V \cap M$ is a border set in $M$, and, being closed, it is nowhere dense. This completes the proof.

Assume additionally that $M$ is a real analytic manifold. Then $V \cap M$ is a real analytic subset of $M$. By Proposition $3, V \cap M$ is nowhere dense in $M$. Therefore we immediately see that $\operatorname{dim}(V \cap M)=k<n$.

Proposition 4. With the above assumptions, $\mathcal{H}_{n}(V \cap M)=0$.

Proof. Since $M$ is compact, therefore in virtue of Lelong's theorem (cf. [8], §18, Prop. 2), $\mathcal{H}_{k}(V \cap M)<\infty$. Now Proposition 1 gives $\mathcal{H}_{n}(V \cap$ $M)=0$. This concludes the proof.

3. Integral representation of the multiplicity. Let $f=\left(f_{1}, \ldots, f_{n}\right)$ : $\left(\mathbb{C}^{n}, 0\right) \rightarrow\left(\mathbb{C}^{n}, 0\right)$, where $n>1$, be a holomorphic mapping with an isolated zero at the point 0 . Then there exists a neighbourhood $G$ of $0 \in \mathbb{C}^{n}$ such that the restriction of $f$ to $G$ is proper (cf. [9], Ch. IV, §1, Prop. 1; [7], Ch. IV, $\S 2$, Prop. 4) and open (cf. [9], Ch. V, §2, Lemma). Assume that $f^{-1}(0) \cap \bar{G}=\{0\}$.

Next, let $D_{j}$ be a disk of the form $\left\{\zeta_{j} \in \mathbb{C}:\left|\zeta_{j}\right|<\varepsilon_{j}\right\}$, and $\Gamma_{j}$ the positively oriented boundary of $D_{j}$, i.e. $\Gamma_{j}$ is the curve with parametric representation $\gamma_{j}\left(t_{j}\right)=\varepsilon_{j} e^{2 \pi i t_{j}}, t_{j} \in[0,1], 1 \leq j \leq n$.

Define $D=D_{1} \times \ldots \times D_{n}$ and $\delta=\Gamma_{1} \times \ldots \times \Gamma_{n}$. Notice that $\bar{D} \subset f(G)$ for sufficiently small numbers $\varepsilon_{j}, 1 \leq j \leq n$. Since $f$ is a proper mapping, $f^{-1}(\bar{D})$ is a compact subset of $G$.

Define $\Pi=\left\{z \in G: f_{j}(z) \in D_{j}, 1 \leq j \leq n\right\}$. Observe that $0 \in \Pi$. As $f(\Pi)=D, \bar{\Pi}$ is a compact subset of $G$. Without loss of generality we 
may assume that $\Pi$ is connected. Then $\Pi$ is a canonical Weil domain, and $\sigma=\left\{z \in G: f_{j}(z) \in \Gamma_{j}, 1 \leq j \leq n\right\}$ is its skeleton (cf. [4], §1).

Let $J_{f}$ be the Jacobian of $f$, and let $G_{0}$ and $\sigma_{0}$ denote the zero sets of $J_{f}$ lying in $G$ and $\sigma$, respectively. Since $f(G)$ is open, $J_{f}$ does not vanish identically. Since $f$ is proper, $f\left(G_{0}\right)$ is an analytic set in $f(G)$ (cf. [9], Ch. V, $\S 5$, Remmert's th.). Moreover, $\operatorname{dim} f\left(G_{0}\right)<n$.

Furthermore, let $\delta^{\prime}$ be the set of points $\zeta=\left(\zeta_{1}, \ldots, \zeta_{n}\right) \in \delta$ such that $\zeta_{j}=\gamma_{j}(0)$ for some $j, 1 \leq j \leq n$. Put $\delta_{0}=f\left(\sigma_{0}\right), \sigma_{0}^{*}=f^{-1}\left(\delta_{0}\right), \sigma^{\prime}=$ $f^{-1}\left(\delta^{\prime}\right)$ and $\tilde{\sigma}=\sigma-\left(\sigma_{0}^{*} \cup \sigma^{\prime}\right)$. Finally, let $\gamma(t)=\left(\gamma_{1}\left(t_{1}\right), \ldots, \gamma_{n}\left(t_{n}\right)\right)$ for $t=\left(t_{1}, \ldots, t_{n}\right) \in[0,1]^{n}$.

LEMMA 1. The set $\delta$ is an $n$-dimensional totally real manifold.

Proof. Consider $\delta$ as a subset of $\mathbb{R}^{2 n}$. Define $g_{j}\left(x_{1}, y_{1}, \ldots, x_{n}, y_{n}\right)=$ $\varepsilon_{j}^{2}-x_{j}^{2}-y_{j}^{2}, 1 \leq j \leq n$. Then $\delta=\left\{p=\left(x_{1}, y_{1}, \ldots, x_{n}, y_{n}\right) \in \mathbb{R}^{2 n}: g_{1}(p)=\right.$ $\left.\ldots=g_{n}(p)=0\right\}$. Since $g_{j}^{\prime}\left(x_{1}, y_{1}, \ldots, x_{n}, y_{n}\right)=\left[0, \ldots, 0,-2 x_{j},-2 y_{j}, 0, \ldots\right.$, $0]$, the differentials $d g_{j}(p)$ are independent for each $p \in \delta$. Thus $\delta$ is an $n$-dimensional real manifold (cf. [11], App. II, Def. 4D).

Fix $p^{0} \in \delta$. Let $w=\left[u_{1}, v_{1}, \ldots, u_{n}, v_{n}\right]$ be a tangent vector to $\delta$ at $p^{0}$. Then $\left(d g_{j}\left(p^{0}\right)\right) w=0$ for each $j, 1 \leq j \leq n$ (cf. [11], App. II, Lemma 5C). This is equivalent to the system of equations $x_{j}^{0} u_{j}+y_{j}^{0} v_{j}=0$ where $p^{0}=$ $\left(x_{1}^{0}, y_{1}^{0}, \ldots, x_{n}^{0}, y_{n}^{0}\right)$. Hence $v_{j}=-u_{j}\left(x_{j}^{0} / y_{j}^{0}\right)$ if $y_{j}^{0} \neq 0$, and $u_{j}=0$ if $y_{j}^{0}=0$, for each $j$. Therefore the $j$ th coordinate of $w$ considered as a vector in $\mathbb{C}^{n}$ is either $\left(u_{j} / y_{j}^{0}\right)\left(y_{j}^{0}-i x_{j}^{0}\right)$ or $i v_{j}$. So, the $j$ th coordinate of $i w$ is either $\left(u_{j} / y_{j}^{0}\right)\left(x_{j}^{0}+i y_{j}^{0}\right)$ or $-v_{j}$. Thus, the latter vector considered in $\mathbb{R}^{2 n}$ has the form $\left[u_{1}^{\prime}, v_{1}^{\prime}, \ldots, u_{n}^{\prime}, v_{n}^{\prime}\right]$ where $u_{j}^{\prime}=u_{j}\left(x_{j}^{0} / y_{j}^{0}\right), v_{j}^{\prime}=u_{j}$ if $y_{j}^{0} \neq 0$, and $u_{j}^{\prime}=-v_{j}, v_{j}^{\prime}=0$ if $y_{j}^{0}=0$. It is easy to see that $T_{p^{0}} \delta \cap i T_{p^{0}} \delta=\{0\}$, so $T_{p^{0}} \delta$ is a totally real subspace in $\mathbb{C}^{n}$. This completes the proof.

Lemma 2. The sets $\gamma^{-1}\left(\delta^{\prime}\right), \gamma^{-1}\left(\delta_{0}\right)$ have Lebesgue measure zero.

Proof. That $\gamma^{-1}\left(\delta^{\prime}\right)$ has measure zero follows immediately from the definitions of $\delta^{\prime}$ and $\gamma$.

Consider now $\gamma^{-1}\left(\delta_{0}\right)$. From the definition we have $\delta_{0}=f\left(\sigma_{0}\right)=f\left(G_{0} \cap\right.$ $\sigma) \subset f\left(G_{0}\right) \cap f(\sigma)=f\left(G_{0}\right) \cap \delta$. Obviously, $\delta$ is compact. So, in virtue of Proposition $3, f\left(G_{0}\right) \cap \delta$ is nowhere dense in $\delta$, while by Proposition 4 , it has $n$-dimensional Hausdorff measure zero. So, by Proposition 1 , it has Lebesgue measure zero. A fortiori, this holds for $\delta_{0}$. From the definition of $\gamma$ it now follows that $\gamma^{-1}\left(\delta_{0}\right)$ has measure zero in $[0,1]^{n}$. This concludes the proof.

Since $f$ restricted to $G \backslash f^{-1}\left(f\left(G_{0}\right)\right)$ is proper and is a local biholomorphism, $f \mid\left(G \backslash f^{-1}\left(f\left(G_{0}\right)\right)\right)$ is a $p$-fold covering. From the assumption that $f^{-1}(0) \cap \bar{G}=\{0\}$ it easily follows that $p=\mu_{c}(f)$ (cf. [9], Ch. V, $\S 7$, Sec. 2). 
Let $h$ be any function holomorphic on $\bar{\Pi}$ and let $\Delta$ be a connected neighbourhood of $\bar{D}$ such that $h$ is defined and bounded on $f^{-1}(\Delta)$.

On $\Delta \backslash f\left(G_{0}\right)$, define

$$
H(\zeta)=\sum_{z \in f^{-1}(\zeta)} h(z) .
$$

Then $H$ is holomorphic and extends to a holomorphic function on the whole $\Delta$ (cf. [4], §2).

In particular, putting $h(z) \equiv 1$, we obtain $H=\mu_{c}(f)$.

From the classical Cauchy formula (cf. [11], Ch. I, §3, (3.6)) we obtain

$$
\frac{1}{(2 \pi i)^{n}} \int_{\delta} \frac{H\left(\zeta_{1}, \ldots, \zeta_{n}\right)}{\zeta_{1} \ldots \zeta_{n}} d \zeta_{1} \ldots d \zeta_{n}=H(0)
$$

where $H(0)=\lim _{\zeta \notin f\left(G_{0}\right), \zeta \rightarrow 0} H(\zeta)$.

With the above notations and assumptions, $\widetilde{\sigma}$ is an $n$-dimensional oriented manifold (cf. [5], Lemma 1.2). So, we may consider the integral

$$
\frac{1}{(2 \pi i)^{n}} \int_{\tilde{\sigma}} h(z) \frac{J_{f}(z)}{f_{1}(z) \ldots f_{n}(z)} d z_{1} \wedge \ldots \wedge d z_{n} .
$$

This integral exists and is equal to (2). We omit the proof of this fact because it runs analogously to the proof of the main theorem in [5].

From the above, for $h(z) \equiv 1$, we have

THEOREM.

$$
\mu_{c}(f)=\frac{1}{(2 \pi i)^{n}} \int_{\tilde{\sigma}} \frac{J_{f}(z)}{f_{1}(z) \ldots f_{n}(z)} d z_{1} \wedge \ldots \wedge d z_{n}
$$

\section{References}

[1] E. M. Chirka, Complex Analytic Sets, Nauka, Moscow 1985 (in Russian).

[2] R. Engelking, General Topology, PWN, Warszawa 1977.

[3] H. Federer, Geometric Measure Theory, Springer, New York 1969.

[4] M. Frontczak, Integral representations of Cauchy type for holomorphic functions on Weil's domains, Bull. Soc. Sci. Łódź, to appear.

[5] - A new simple proof of Weil's integral formula for canonical domains, ibid., to appear.

[6] P. Griffiths and J. Harris, Principles of Algebraic Geometry, Wiley-Interscience, New York 1978.

[7] M. Hervé, Several Complex Variables, Local Theory, Oxford Univ. Press, Bombay 1963.

[8] S. Lojasiewicz, Ensembles semi-analytiques, I.H.E.S., Bures-sur-Yvette 1965.

[9] - Introduction to Complex Analytic Geometry, PWN, Warszawa 1988 (in Polish). 
[10] B. V. Shabat, Introduction to Complex Analysis, Part II, Nauka, Moscow 1985 (in Russian).

[11] H. Whitney, Complex Analytic Varieties, Addison-Wesley, Reading, Mass., 1972.

INSTITUTE OF MATHEMATICS

ŁÓDŹ UNIVERSITY

BANACHA 22

90-238 ŁÓDŹ, POLAND

Reçu par la Rédaction le 12.9.1990 